\title{
How to Improve the Clinical Development Paradigm and its Division into Phases I, II and III
}

\author{
Marion Bamberger ${ }^{1}$, Nicholas Moore ${ }^{2}$, Philippe Lechat ${ }^{3}$ and the participants of Round Table $N^{\circ} 3$ of Giens XXVI \\ 1 Laboratoire Bristol Myers Squibb, Rueil Malmaison, France \\ 2 Service de Pharmacologie, Université Victor Segalen, Bordeaux, France \\ 3 Afssaps, Saint Denis, France
}

Text received may 9, 2011; accepted may 19, 2011

\author{
Keywords: \\ clinical drug \\ development; \\ clinical research; \\ phase I; \\ phase II; \\ phase III; \\ phase IV; \\ regulatory; \\ adaptative design
}

\begin{abstract}
Based on the observation that over the last 30 years the cost of development has risen regularly as the number of new chemical entities reaching the market has fallen, how can "savings" be made in terms of clinical development, the objective being more rapid access to a drug for medical needs that are not covered? Several instruments exist to enable innovative products to be made available more quickly: temporary use authorisations, which are not concerned by this work (ATUs), conditional marketing authorisations (MAs) and MAs under exceptional circumstances.

These aspects have been taken up in the European medicines agency (EMA)'s "Road Map", which states "A key issue for Regulators will be if a more "staggered" approval should be envisaged, characterised by a better defined/more restricted population of good responders, followed by a broadening of the population post-authorisation when more "real life" data are available. In addition, maximising the value of information generated in the post-authorisation phase should be developed through the use of cohorts and other prospectively collected use data, especially in the case of conditional marketing authorisations."

The rules of procedure of the Transparency Commission for their part provide for the notion of preliminary examination: in order to prepare as best as possible the examination of dossiers of products assumed to be innovative and to limit delays, the office can undertake a preliminary study as soon as the dossier has been filed at the Committee for medicinal products for human use (CHMP). It may, at this time, request the firm to provide further information and may call on external experts. The implementation of this preliminary study does not exonerate the firm of the obligation of filing a complete dossier. The post inscription studies requested by the Transparency Commission (ISPEP - public health benefit and post-marketing studies) are usually requested in the case of hesitations regarding the level of improvement of the medical benefit (ASMR) [level II/III or IV/V]. Such requests mainly concern uncertainties regarding the transposability, the patient profile or correct usage in real life. Among the studies whose results were provided, in 15 cases the results were in line with expectations, in 6 cases they resulted in downward re-evaluations and the final 3 cases were inconclusive.

The final recommendations of the round table were:

- Defining the medical need that is not covered by working in consultation (Industry and Health Authorities);

- Providing a Complementary Investigations Plan (PIC) after the MA at a very early stage to reinforce the early MA, and/or HTA (health technology assessment) preparation and monitoring (possible constraining actions);

- Enhanced use of modelling techniques and their transposability;

- "Intussusception" of phases to optimise the development of a complete dossier;

- Early "scientific opinions" (EMA, French Health Products Safety Agency [Afssaps], French Health Authority [HAS]);

- Raising the awareness of the authorities, industry, doctors and patients with regard to controlled observational studies;

- Developing the use of public data bases.
\end{abstract}

\footnotetext{
* For the list of participants, see end of article
} 


\section{Purpose of the Round Table}

Based on the observation that, for the last 30 years, the cost of development has been regularly increasing, while the number of new chemical entities reaching the market has been decreasing, the objective of this Round Table was to propose measures designed to achieve "savings" in terms of clinical development while ensuring more rapid access to medicinal products to cover as yet unmet medical needs.

\section{Current situation}

Several instruments are available to allow more rapid availability of innovative products:

- ATU (early access programs, which are not concerned by this study);

- Marketing authorisations (MA) under exceptional circumstances correspond to cases in which some studies under normal conditions of use are lacking (efficacy and safety of use data), when the marketing authorisation holder justifies a rare indication or a small target population, lack of available information in the current state of scientific knowledge, and when ethical considerations make it difficult to collect missing data. A scientific opinion must be requested 4 to 6 months before the submission. Specific measures can be requested by regulatory authorities: risk management plan, post-marketing studies, as well as a mention in the summary of product characteristics (SPC) and in the patient information leaflet (PIL) about the nature of the MA. Particular conditions of use or dispensing may also be requested;

- Conditional marketing authorisations, which are granted under 4 conditions: a favourable benefit-risk balance (based on substitution criteria, in special populations, or on interim analyses), clinical data to be completed (particularly in special populations), presence of an unmet medical need, and when the public health benefit outweighs the risks, justifying access to the medicinal product before completion of the usual MA application. However, all regulatory data concerning quality and non-clinical data must be available (except in emergency situations) and the conditional nature of the MA must be indicated in the SPC and the patient information leaflet. This MA is granted for 1 year, renewable.

These elements are summarised in the EMA "road map", which states: "A key issue for Regulators will be if a more "staggered" approval should be envisaged, characterised by a better defined/more restricted population of good responders, followed by a broadening of the population post-authorisation when more "real life" data are available. In addition, maximising the value of information generated in the post-authorisation phase should be developed through the use of cohorts and other prospectively collected use data, especially in the case of conditional marketing authorisations."

The internal regulations of the Transparency Commission propose the concept of preliminary examination: to ensure optimal evaluation of submissions for products considered to be innovative and to limit delays, the executive committee can conduct a preliminary study immediately after filing of the submission to the committee for medicinal products for human use (CHMP). The executive committee may ask the applicant to provide complementary information and call on external experts. This preliminary evaluation does not eliminate the need to file a complete application.

Post-marketing studies are often requested by the Transparency commission when there is a doubt about the level of improvement of the relative medical benefit provided (relative medical benefit or ASMR; level II/III or IV/V). These requests essentially concern uncertainties about transposability, the patient profile or compliance with good use under real life conditions. Among the studies for which the results are available, the results were in line with expectations in 15 cases, the results led to a decrease of the ASMR provided in 6 cases, and the results were inconclusive in 3 cases.

\section{Round Table proposals}

Under these conditions, the round table issues proposals or lines of reflection on measures designed to achieve the objectives of acceleration and simplification of clinical development.

Some of these strategies are general and apply to all types of marketing authorisation applications:

- Extend phase I studies to patients (phase I/II);

- Develop comparative phase II studies (Phase II-III); ${ }^{[1]}$

- Optimize the choice of doses by modelling, or by more precisely determining the biologically effective dose;

- Use validated biomarkers; ${ }^{[2]}$

- Finally, evaluate the possibility to use adaptive clinical trial methods ${ }^{[3-17]}$ by including in the protocol continuation of open-label follow-up of patients in whom the trial was prematurely terminate because of efficacy, in order to complete safety data.

Other strategies must be adapted to particular situations:

- For example, in the case of a major unmet medical need, marketing authorisation may be granted more rapidly on the basis of limited data, but with optimized development, and a complementary investigation plan (CIP) to be developed after marketing; 
- In the case of the first molecule of a new class, a "classical" marketing authorisation application with the use of facilitating strategies, such as adaptive methods, optimized use of PK/PD data;

- In the case of the third or subsequent member of a class that is already marketed and well known, applicants should be able to take advantage of the experience acquired with this class: does all of the development need to be repeated, particularly concerning efficacy in the fields covered by validated biomarkers? Abridged development on certain aspects does not eliminate the need for strict evaluation of the safety of each product which can be different from that of the class; initiation and participation in class post-inscription studies (studies by disease) can also be proposed, by carefully developing methods of inclusion of new products in ongoing studies as they are released onto the market. This approach could be simpler in epidemiological studies by disease than in the case of ad hoc cohorts based on product exposure;

- In case of new indications for a product that is already marketed:

- When the molecular target remains the same, for example nonspecific growth factors (VEGF) in various types of cancers: a proof of concept study must be set up and available data can be used;

- When a new indication is targeted with similar target population characteristics, pre-existing safety data could be taken into account; (e.g. development of a targeted anticancer drug in another type of cancer);

- When the target population characteristics are different, solid safety data must be obtained in this new target population (e.g. use of targeted therapies in non-cancer populations).

\section{Round Table's final recommendations}

The Round Table's final recommendations were as follows:

- The definition of unmet medical needs must be pursued by consultation between drug companies and health authorities, and unmet medical needs must be identified in order to anticipate possibilities for facilitated or accelerated development;

- A post-marketing complementary investigation plan (CIP) must be set up as early as possible in support of a fast-track marketing authorisation;

- Prepare and monitor evaluation of the added value of the product (health technology assessment or transparency) that may lead to certain regulatory measures before or after marketing;

- Increased use of modelling techniques and their transposability to the post-marketing real use setting;
- "Intussusception" or integration of successive phases of clinical development in order to optimize development of a complete Marketing Authorisation application;

- Early "scientific opinions" (European medicines agency (EMA), Agence française de sécurité sanitaire des produits de santé (Afssaps), Haute autorité de santé (HAS)) ;

- Increase the awareness of authorities, manufacturers, doctors and patients about the importance of controlled observational studies;

- Develop the use of public databases.

\section{Participants}

Michel Azizi (CIC, HEGP Paris), Patrick Blin (Pharmacologie, Bordeaux), Myriam Bouhassira (Lilly France), Dominic Cellier (Merck Serono), Jean-Paul Demarez (Pierre Fabre Médicaments), Xavier Duval (Inserm AP-HP Paris), François Gueyffier (CHU Lyon), Claire Le Jeunne (Hotel Dieu, Paris), Christian Libersa (CIC, CHRU Lille), Florence Mahlberg-Gaudin (Servier), Patrick Maison (CHU Henri Mondor, Paris), Pierre Marquet (Hôpital Dupuytren, Limoges), Mathieu Molimard (CHU, Bordeaux), Aurélie Moser (Roche), Mira Pavlovic (HAS), Pascal Piedbois (Astra Zeneca), Odile Regnier (CNAMTS), Jean-Charles Reynier (AP-HM, Marseille), Catherine Rey-Quinio (Afssaps), Patrick Rossignol (CIC, CHU Vandoeuvre les Nancy), Alain Spriet (Alain Spriet Conseil), Franck Vignal (Sanofi Aventis).

\section{References}

1. Jenkins M, Stone A, Jennison C. An adaptive seamless phase II/III design for oncology trials with subpopulation selection using correlated survival endpointsdagger. Pharm Stat 2010 Dec 8

2. Eickhoff JC, Kim K, Beach J, et al. A Bayesian adaptive design with biomarkers for targeted therapies. Clin Trials 2010 Oct; 7(5): 546-56

3. Benda N, Brannath W, Bretz F, et al. Perspectives on the use of adaptive designs in clinical trials. Part II. Panel discussion. J Biopharm Stat 2010 Nov; 20(6): 1098-112

4. Brannath W, Burger HU, Glimm E, et al. Comments on the draft guidance on "adaptive design clinical trials for drugs and biologics" of the U.S. Food and Drug Administration. J Biopharm Stat 2010 Nov; 20(6): 1125-31

5. Chang M. Introduction to discussion papers on draft FDA guidance on adaptive designs. J Biopharm Stat 2010 Nov; 20(6): 1113-4

6. Cheng B, Chow SC. On flexibility of adaptive designs and criteria for choosing a good one-a discussion of FDA draft guidance. J Biopharm Stat 2010 Nov; 20(6): 1171-7

7. Chow SC. A note on special articles on adaptive clinical trial designs. J Biopharm Stat 2010 Nov; 20(6): 1088-9

8. Chuang-Stein C, Beltangady M. FDA draft guidance on adaptive design clinical trials: Pfizer's perspective. J Biopharm Stat 2010 Nov; 20(6): 1143-9

9. Cook T, DeMets DL. Review of draft FDA adaptive design guidance. J Biopharm Stat 2010 Nov; 20(6): 1132-42

10. Emerson SS, Fleming TR. Adaptive methods: telling "the rest of the story". J Biopharm Stat 2010 Nov; 20(6): 1150-65 
11. Fu H, Manner D. Bayesian adaptive dose-finding studies with delayed responses. J Biopharm Stat 2010 Sep; 20(5): 1055-70

12. Gallo P, Anderson K, Chuang-Stein C, et al. Viewpoints on the FDA draft adaptive designs guidance from the PhRMA working group. J Biopharm Stat 2010 Nov; 20(6): 1115-24

13. Liu Q, Chi GY. Understanding the FDA guidance on adaptive designs: historical, legal, and statistical perspectives. J Biopharm Stat 2010 Nov; 20(6): 1178-219

14. Lu Q, Tse SK, Chow SC. Analysis of time-to-event data under a two-stage survival adaptive design in clinical trials. J Biopharm Stat 2010 Jul; 20(4): 705-19

15. Wang SJ. Editorial. Adaptive designs: appealing in development of therapeutics, and where do controversies lie? J Biopharm Stat 2010 Nov; 20(6): 1083-7
16. Wang SJ. Perspectives on the use of adaptive designs in clinical trials. Part I. Statistical considerations and issues. J Biopharm Stat 2010 Nov; 20(6): 1090-7

17. Zhou Y. Adaptive designs for Phase I dose-finding studies. Fundam Clin Pharmacol 2010 Apr; 24(2): 129-38

Correspondence and offprints: Nicholas Moore, Service de Pharmacologie, Université Victor Segalen, Zone Nord, Bat 1A, CHU de Bordeaux, 33076 Bordeaux Cedex, France.

E-mail: nicholas.moore@pharmaco.u-bordeaux2.fr 